\title{
Revealing the effects of temporal orienting of attention on response conflict using continuous movements
}

\author{
Melisa Menceloglu ${ }^{1,2}$ (D) Satoru Suzuki ${ }^{2,3} \cdot$ Joo-Hyun Song ${ }^{1,4}$
}

Accepted: 22 December 2020 / Published online: 13 January 2021

(C) The Psychonomic Society, Inc. 2021

\begin{abstract}
Orienting attention in time enables us to prepare for forthcoming perception and action (e.g., estimating the duration of a yellow traffic light when driving). While temporal orienting can facilitate performance on simple tasks, its influence on complex tasks involving response conflict is unclear. Here, we adapted the flanker paradigm to a choice-reaching task where participants used a computer mouse to reach to the left or right side of the screen, as indicated by the central arrow presented with either the congruent or incongruent flankers. We assessed the effects of temporal orienting by manipulating goal-driven temporal expectation (using probabilistic variations in target timing) and stimulus-driven temporal priming (using sequential repetitions versus switches in target timing). We tested how temporal orienting influenced the dynamics of response conflict resolution. Recent choice-reaching studies have indicated that under response conflict, delayed movement initiation captures the response threshold adjustment process, whereas increased curvature toward the incorrect response captures the degree of coactivation of the response alternatives during the controlled response selection process. Both temporal expectation and priming reduced the initiation latency regardless of response conflict, suggesting that both lowered response thresholds independently of response conflict. Notably, temporal expectation, but not temporal priming, increased the curvature toward the incorrect response on incongruent trials. These results suggest that temporal orienting generally increases motor preparedness, but goal-driven temporal orienting particularly interferes with response conflict resolution, likely through its influence on response thresholds. Overall, our study highlights the interplay between temporal orienting and cognitive control in goal-directed action.
\end{abstract}

Keywords Temporal processing $\cdot$ Perception and action $\cdot$ Goal-directed movements

Just as we can orient attention in space and to certain object features, recent research has shown that we can also orient attention in time (for reviews, see Nobre et al., 2007; Nobre $\&$ van Ede, 2018). Temporal orienting enables us to efficiently prepare for forthcoming perception and action in many everyday situations - for instance, when estimating the duration of a yellow traffic light when driving, approximating when to

Melisa Menceloglu

melisa_menceloglu@brown.edu

1 Department of Cognitive, Linguistic \& Psychological Sciences, Brown University, 190 Thayer St, Providence, RI 02912, USA

2 Department of Psychology, Northwestern University, Evanston, IL, USA

3 Interdepartmental Neuroscience Program, Northwestern University, Evanston, IL, USA

4 Carney Institute for Brain Science, Brown University, Providence, RI, USA swing at a tennis ball, or deciding when to jump into a conversation. Temporal orienting can be generated by goal-driven biases - such as by knowledge of when to expect a target, akin to cueing (temporal expectation). In laboratory tasks, when a target appears with expected timing compared with unexpected timing or when no temporal expectation is implied, responses are usually faster and/or more accurate (e.g., Correa et al., 2005; Coull \& Nobre, 1998; Rohenkohl et al., 2012; Vangkilde et al., 2012). Temporal orienting can also be driven by stimulus-driven or history-driven biases - such as by recent target-timing history, akin to repetition priming (temporal priming; also referred to as sequential effects). For instance, when a target onset timing is repeated instead of switched across trials, responses are faster (e.g., Los, 2010; Los \& Van den Heuvel, 2001; Steinborn et al., 2008). The presence of distinct goal-driven and stimulus/history-driven temporal orienting mechanisms has been shown by their dissociable effects such as temporal expectation, but not priming, being affected by a secondary task (Correa et al., 2004) and recruiting prefrontal structures (Triviño et al., 2010), as well 
as their unique effects on behavioral performance when concurrently present (e.g., Los \& Van den Heuvel, 2001; Menceloglu et al., 2019).

Many daily activities that involve temporal orienting and precise temporal coordination between perception and action (e.g., driving) also involve competing sensory information and response alternatives. For instance, when waiting for the red traffic light to turn green, to go straight ahead at an intersection, the onset of a green left-turn light while the straightahead light is still red creates a response conflict. One can imagine that while increased preparation facilitates a timely start to driving when there are only straight-ahead lights, it may cause hasty decisions when there is conflicting information. Here, we asked, While temporal orienting can facilitate behavioral performance in simple tasks, how does it influence performance in more complex tasks involving competing response choices? This question is still outstanding as only a few studies have examined such effects and revealed heterogeneous results.

Focusing on temporal expectation and using choice response times (RTs), some researchers have shown null effects of temporal orienting on response conflict (Ball et al., 2019; Menceloglu et al., 2017), whereas others have reported that temporal expectation could interfere with response conflict resolution, albeit with relatively small effects (e.g., Correa et al., 2010; Korolczuk et al., 2018). In particular, Correa et al. (2010) found that fulfilled temporal expectations increased the Eriksen flanker and Simon effects (Eriksen \& Eriksen, 1974; Simon \& Wolf, 1963) on key-press RT. Korolczuk et al. (2018) further found that having $100 \%$ valid temporal cues predicting short or long cue-to-target intervals increased the Simon conflict effect on key-press RT. Combined with the facilitatory effects of temporal expectation on overall response speed, these findings suggest that temporal expectation may increase general motor readiness, which can then interfere with response conflict resolution, a process that requires cautious rather than hasty responses. Despite the recent interest in the effects of temporal expectation on response conflict, to our knowledge, no one has reported either the presence or absence of temporal priming effects on response conflict resolution.

We therefore aimed to better understand how goaldriven as well as stimulus-driven temporal orienting mechanisms might influence response conflict within the same experimental paradigm. To achieve this goal, we generated temporal orienting endogenously by the knowledge of target timing probability in a block of trials - temporal expectation-and exogenously by local repetitions or switches of target timing across trials-temporal priming (e.g., Menceloglu et al., 2019; Mento, 2017) within a flanker task. Further, we adapted the flanker paradigm to a choice-reaching task where participants used a computer mouse to reach to the left or the right side of the screen, as indicated by the central arrow presented with either the congruent or incongruent flanker arrows.

Prior studies have consistently shown that reach trajectories obtained in a choice-reaching task provide a unique tool to observe changes in internal cognitive decisions unfolding over time; across various populations, including children, older adults, and nonhuman primates; and with devices such as a three-dimensional reach tracker, stylus, or computer mouse (e.g., Erb \& Marcovitch, 2018; Erb et al., 2016; Erb et al., 2017; Incera \& McLennan, 2018; Faulkenberry, Cruise, Lavro, \& Shaki, 2016; Moher \& Song, 2019; Scherbaum et al., 2010; Spivey et al., 2005;). The analysis of continuous motor output, unlike discrete key-press responses, can provide unique insights into cognition as action and cognition are integrated rather than discrete processes (for reviews, see Dotan et al., 2019; Song, 2017; Song \& Nakayama, 2009).

A recent series of work has suggested that choice-reaching tasks can dissociate processes underlying cognitive control while RTs from conventional key-press tasks can only capture the combination of these processes as a whole (Erb \& Marcovitch, 2018; Erb et al., 2016; Erb et al., 2017). The current representative cognitive control framework (Ridderinkhof et al., 1995; Ridderinkhof et al., 2020; Shenhav et al., 2013) posits a set of general processes involved in response conflict resolution. When conflict occurs on incongruent trials, both the correct and the incorrect response alternatives are activated via the control-demanding and automatic pathways, respectively. The monitoring process registers this coactivation of the competing responses. Then, in response to this, the response threshold adjustment process slows down the motor output, which is then followed by the controlled response selection process, in which the activation along the control-demanding pathway is increased via top-down control to bias the response activation in favor of the correct response (see Erb \& Marcovitch, 2018, for a clear illustration of the response threshold adjustment process and the controlled response selection process).

Specifically, Erb and colleagues (Erb \& Marcovitch, 2018; Erb et al., 2016; Erb et al., 2017; Erb et al., 2020) suggested that the movement initiation latency (the time between stimulus onset and movement onset) reflects the response threshold adjustment process while movement curvature (the amount of deviation in the reach trajectory from the direct path to the target) reflects the controlled response selection process. This idea is supported by a few findings. First, initiation latency is longer on incongruent than on congruent trials, and the trajectories are curved toward the incorrect response alternative on incongruent trials, whereas there is little or no curvature (no bias) on congruent trials. Second, the effects of congruency on initiation latency and curvature are differently modulated in various contexts - for instance, by trial-sequence effects including the congruency of the previous trials and 
response (e.g., left or right; Erb \& Marcovitch, 2018; Erb et al., 2016; Erb et al., 2017; Erb et al., 2020).

Therefore, the current study with a choice-reaching task enabled us to determine whether temporal orienting influences response conflict resolution, and if so, whether it modulates the response threshold adjustment process, the controlled response selection process, or both. For instance, we reasoned that if temporal orienting selectively modulates the response threshold adjustment during the flanker task, we will observe an increased congruency effect on initiation latency while maintaining the congruency effect on the magnitude of curvature. In contrast, if temporal orienting selectively affects the controlled response selection process, we will observe the opposite. We also determined how temporal orienting induced by temporal expectation and temporal priming might differently influence response conflict resolution. Finally, by analyzing the correlation between initiation latency and the magnitude of curvature, we determined a flexibly compensatory relation between the response threshold adjustment and controlled response selection processes.

\section{Method}

\section{Participants}

Eighteen Northwestern University undergraduate students were recruited to participate in the study. All were righthanded and had normal or corrected-to-normal vision. Participants gave informed consent and were treated according to the guidelines of the Institutional Review Board at Northwestern University. Participants received partial course credit for their participation, which lasted approximately 1 hour. Our sample included all 18 participants (nine women, nine men) between the ages of 18 and 21 years $(\mathrm{M}=18.56$ years, $\mathrm{SD}=0.92$ ).

\section{Stimuli and procedure}

Stimuli were presented using a 13-inch, $2.3 \mathrm{GHz}$ MacBook Pro, running MATLAB (Version R2016b) with Psychtoolbox extensions (Version 3.0.14; Brainard, 1997; Kleiner et al., 2007; Pelli, 1997). All visual stimuli were black $\left(1.2 \mathrm{~cd} / \mathrm{m}^{2}\right)$ on a light-gray background $\left(73 \mathrm{~cd} / \mathrm{m}^{2}\right)$. The viewing distance was $\sim 60 \mathrm{~cm}$.

The experimental design and sequences of trial events are illustrated in Fig. 1. Each trial began with the "ready" display, consisting of three empty circles serving as placeholders for the start location and the two response locations. The startlocation placeholder $\left(0.57^{\circ}\right.$ visual angle diameter $)$ was placed at $4.64^{\circ}$ of visual angle from the bottom of the screen (measured from the center of the circle) and horizontally centered. The two response-location placeholders $\left(1.13^{\circ}\right.$ visual angle diameter) were placed at $4.64^{\circ}$ of visual angle from the top of the screen (measured from the center of the circle) and horizontally off-centered by $\pm 4.25^{\circ}$ of visual angle. The ready display was presented until the participants clicked within the start-location placeholder. Once the participants clicked within the start location using an external, wireless mouse with their right hand, the interval display was immediately presented, which included minor changes to the ready display; a fixation cross $\left(0.47^{\circ} \times 0.47^{\circ}\right.$ visual angle $)$ appeared between the response-location placeholders, and the start-location placeholder was replaced with a filled-in circle. The position of the cursor was accentuated by presenting a black filled-in circle $\left(0.57^{\circ}\right.$ visual angle diameter) throughout the trial.

We generated temporal expectation by block-wise manipulating the probability of the interval duration, which could be short $(0.6 \mathrm{~s})$, medium $(1.2 \mathrm{~s})$, or long $(2.4 \mathrm{~s})$. In the short-fixed block, only the $0.6 \mathrm{~s}$ interval was used; in the long-fixed block, only the $2.4 \mathrm{~s}$ interval was used; and in the mixed blocks, the three intervals were equiprobable. Participants were required to keep the cursor within the start-location placeholder during the interval display; otherwise, a warning message appeared, and the trial was sent back to the ready display.

Immediately following the interval display, the target display of five arrows in a row $\left(4.34^{\circ} \times 0.75^{\circ}\right.$ visual angle $)$ consisting of a central target arrow and four "flanker" arrows was presented. The flanker arrows could point to the same direction (congruent condition) or the opposite direction (incongruent condition) as the central arrow, with equal probability. Participants were instructed to move the cursor to the response location to which the center arrow pointed as quickly and as accurately as possible within $1 \mathrm{~s}$-otherwise, the trial was classified as incorrect.

At the end of the response window, an auditory feedback was provided (a $100 \mathrm{~ms} 600 \mathrm{~Hz}$ pure tone for correct responses and $300 \mathrm{~Hz}$ tone for incorrect responses) using the computer speakers $(\sim 60 \mathrm{~dB})$. Next, the ready display was immediately presented for the next trial.

We divided the experiment into five blocks of trials - one short-fixed block, one long-fixed block, and three mixed blocks - with a total of 590 trials. Within each block, an equal number of congruent and incongruent flanker trials, and in parallel, an equal number of left and right trials, were presented in a randomized order. The first 10 trials in each block served as practice trials and were excluded from analysis, leaving 540 experimental trials in total. Note that we consecutively repeated the mixed block three times to equate the number of trials for the short and long intervals between the fixed-interval and mixed-interval conditions. The order of the short-fixed block, long-fixed block, and the three mixed blocks was counterbalanced in that six participants were included in each of the three counterbalancing cells, completing the blocks in either one of the three orders: (1) short-mix-long; (2) mix-long-short; (3) long-short-mix. We gave participants a 


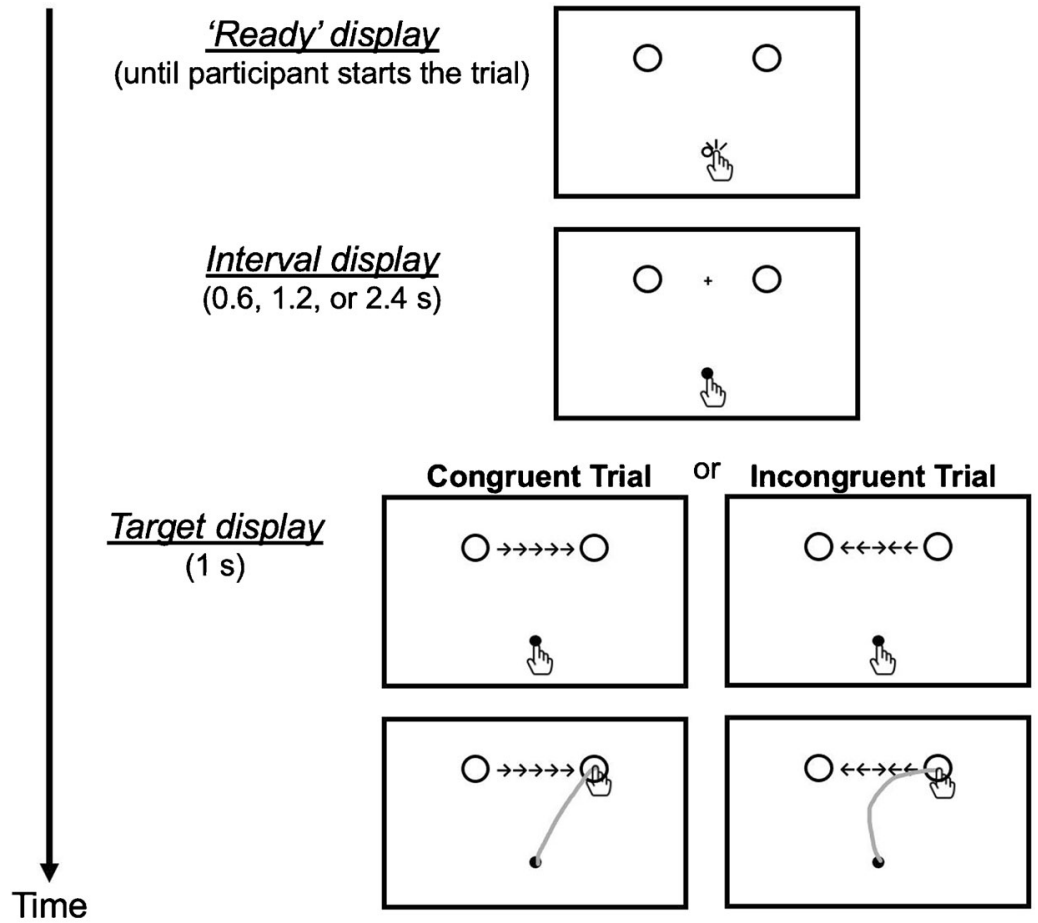

Fig. 1 Experimental design and sequences of trial events. Each trial started with the "Ready" display. Participants clicked on the startlocation placeholder to proceed with the trial. Then, the interval display was presented for $0.6,1.2$, or $2.4 \mathrm{~s}$ with different probabilities depending on the block. Participants needed to hold the cursor within the startlocation placeholder until the target was presented. Upon the presentation of the arrows, participants' task was to move the cursor to the response-

brief break between the blocks (i.e., every 108 trials). Participants were informed of the probabilities of the fixed and varied pretarget intervals before each block.

\section{Data analysis}

The data analysis methods used in the current study closely followed those from Moher and Song (2013). We recorded the $x$ and $y$ coordinates of the cursor with a $60-\mathrm{Hz}$ sampling rate; we processed and analyzed the data using MATLAB and R. The raw data were filtered using a second order, low-pass Butterworth filter with a cutoff of 10 $\mathrm{Hz}$.

We defined movement onset as the first time point on each trial after stimulus onset at which cursor movement's speed exceeded $1 \mathrm{~cm} / \mathrm{s}$; the speed was calculated as the linear distance traveled between successive cursor-position samples (i.e., the square root of the sum of the squared distance traveled along the $x$ and $y$ axes) divided by the intersample interval. We defined movement offset as the first measurement on each trial when the speed decreased to below $1 \mathrm{~cm} / \mathrm{s}$. Individual trials were inspected to assure that the $1-\mathrm{cm} / \mathrm{s}$ criterion properly captured movement onset and offset. For trials location placeholder to which the central arrow pointed as quickly as possible, within the 1-s window. The cursor position was indicated with a small black dot throughout the trial; neither the hand cursor icon nor the continuous cursor trajectory shown in gray were presented in the actual experiment. The gray curves presented here demonstrate the hypothesized effects of flanker congruency on cursor trajectories

in which this criterion clearly missed part of the movement, thresholds were manually adjusted for that trial $(<1 \%$ of all trials).

To characterize the chronometric aspects of reach movements, we defined initiation latency as the time elapsed between stimulus onset and movement onset, movement time as the time elapsed between movement onset and movement offset, and total time as the sum of initiation latency and movement time.

To quantify the reach movement trajectories, we first resampled each movement to 101 points equally spaced in $y$ dimension (corresponding to the vertical dimension of computer screen) for comparison using the normalization methods described in detail in Gallivan and Chapman (2014). Then, using the resampled data, we characterized the global movement trajectory by computing curvature and a distractor attraction score.

We measured curvature in two-dimensional space by calculating a line from the start to the end point of the movement and measuring the orthogonal deviation of the actual movement from that line at each sample throughout the movement. We defined curvature as the signed maximum point of deviation in millimeters (e.g., Desmurget et al., 1997; Erb et al., 
2016; Erb et al., 2017; Moher \& Song 2013; Song \& Nakayama, 2006). Positive values indicated the degree of curvature away from the straight line and toward the incorrect response side, negative values indicated curvature away from the straight line and toward the correct response side, and a value of zero indicated an unbiased, straight-line trajectory.

To generate distractor attraction scores (Erb et al., 2016; Erb et al., 2017; Moher, Anderson, et al., 2015; Moher, Sit, et al., 2015) for each participant, we calculated the average trajectory for each combination of temporal orienting (separately for temporal expectation and temporal priming conditions) and flanker congruency. Then, within each temporal orienting condition, at each of the 101 movement points, we calculated the distance between the positions of the cursor for two averaged trajectories from congruent and incongruent flanker conditions (incongruent minus congruent difference trajectory). The resultant distance was calculated as positive if the cursor was pulled closer to the side of the incorrect response alternative.

We measured temporal expectation effects as the differences in the abovementioned behavioral outcomes between the short-interval trials in the short-fixed block, when a target was presented with the short interval $100 \%$ of the time- the strong-temporal-expectation condition-relative to the shortinterval trials in the mixed block, when a target was presented with the short interval $33 \%$ of the time - the weak-temporalexpectation condition. We focused only on the short-interval trials for analyses because the long-interval trials do not have the same strong versus weak temporal expectation distinction, as participants can reorient their attention to the long interval upon not receiving the target after the short or medium interval in the mixed block (Correa et al., 2004; Coull \& Nobre, 1998; see the Supplementary Material for the results on the longinterval trials). Next, using the mixed block trials, we measured temporal priming effects as the differences in the behavioral outcomes between when a given trial was preceded by a same-timing trial (e.g., a short-interval trial preceded by a short-interval trial) - the primed-timing condition - and when it was preceded by a different-timing trial (e.g., a shortinterval trial preceded by a medium-interval or long-interval trial) - the unprimed-timing condition. Similarly, we focused on the current short-interval trials, as typical temporal priming effects are asymmetric in that they are absent for current longer-interval trials (e.g., Los \& Van den Heuvel, 2001; Vallesi \& Shallice, 2007). Lastly, we measured response conflict effects as the differences between the congruent condition and the incongruent condition.

In particular, to test the effects of temporal expectation on response conflict, we conducted a $2 \times 2$ repeatedmeasures analysis of variance (ANOVA) with factors temporal expectation (strong vs. weak) and congruency (congruent vs. incongruent) separately on initiation latency, movement time, total time, and curvature. We also tested the effects of temporal expectation on distractor attraction score using a repeated-measures $t$ test, correcting for multiple comparisons for 101 points. We then tested the effects of temporal priming on response conflict using the same set of analyses with the factors temporal priming (primed vs. unprimed) and congruency. In all of the analyses, we used data from correct trials (i.e., trials on which participants reached the correct response location within the 1-s response window) and short-interval trials.

\section{Results}

Here, we separately examined the effects of temporal expectation and temporal priming on response conflict.

\section{Temporal expectation interferes with response conflict resolution}

Evidence from chronometric measures: Initiation latency, movement time, and total time On average, task accuracy was relatively high $(M=89.12 \%, S E=2.49 \%)$ and the majority of the errors $(\sim 70 \%)$ were due to missing the time deadline. Participants were more accurate on the strong-temporalexpectation $(\mathrm{M}=90.94 \%, \mathrm{SE}=2.56 \%)$ than on the weaktemporal-expectation trials $(M=87.29 \%, \mathrm{SE}=2.64 \%), F(1$, 17) $=7.98, p=.012, \eta_{p}{ }^{2}=0.32$, as well as on the congruent $(\mathrm{M}$ $=91.31 \%, \mathrm{SE}=2.04 \%)$ than incongruent trials $(\mathrm{M}=86.93 \%$, $\mathrm{SE}=3.07 \%), F(1,17)=5.61, p=.03, \eta_{\mathrm{p}}{ }^{2}=0.25$, with no significant interaction between temporal expectation and congruency on accuracy, $F(1,17)=7.98, p>.30$. This provides no evidence of a speed-accuracy trade-off when the initiation latency effects are considered.

We then analyzed initiation latencies (see Fig. 2a), measured as the time between stimulus onset and movement onset, which capture the threshold adjustment process (Erb \& Marcovitch, 2018; Erb et al., 2016; Erb et al., 2017). As predicted, participants initiated their movement later when flankers were incongruent relative to congruent. This confirms that response thresholds are elevated in the presence of response conflict. In parallel, participants initiated their movement earlier when they had strong relative to weak temporal expectation about the target onset, replicating previous key-press findings that response thresholds are reduced with temporal orienting. Notably, these effects did not interact, pointing to adjustments to response threshold via independent mechanisms. This pattern is confirmed by an ANOVA with a significant main effect of congruency, $F(1,17)=13.93, p=.002, \eta_{\mathrm{p}}{ }^{2}=$ 0.45 , a significant main effect of temporal expectation, $F(1,17)=66.09, p<.0001, \eta_{\mathrm{p}}{ }^{2}=0.80$, and no interaction effect between them, $F(1,17)=3.40, p>.08$ (see Fig. $2 \mathrm{a})$. These results suggest that response conflict elevates 


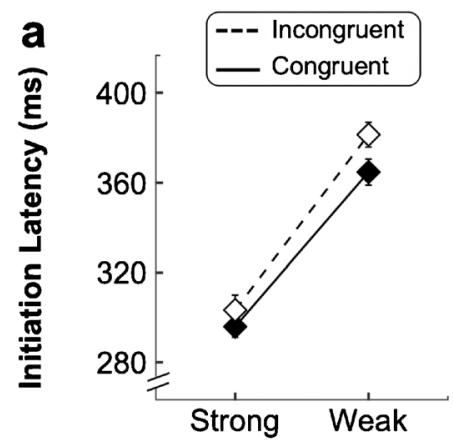

Temporal Expectation

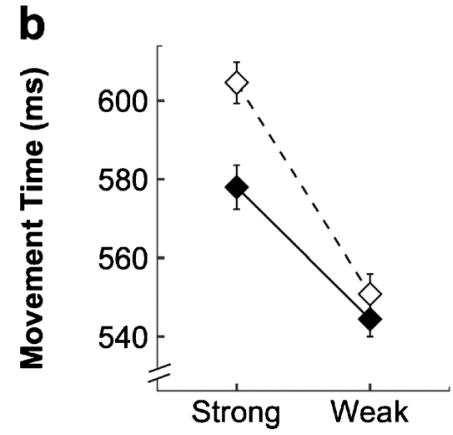

Temporal Expectation

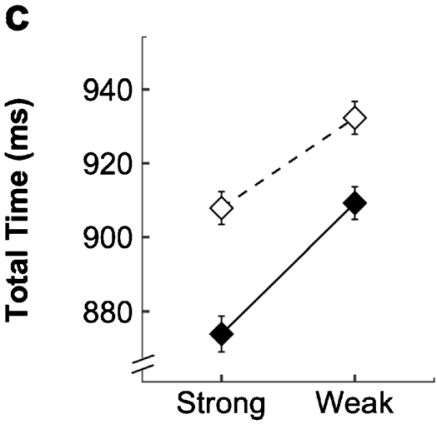

Temporal Expectation

Fig. 2 The effects of temporal expectation and flanker congruency on (a) initiation latency, (b) movement time, and (c) total time are shown. The error bars represent \pm 1 standard error of the mean, adjusted for within-participants comparisons (Morey, 2008)

response threshold and temporal expectation lowers response threshold independently of one another.

Next, we analyzed movement times (see Fig. 2b), measured as the time between movement onset and movement offset. We observed that participants took longer to complete the reach movement when flankers were incongruent relative to congruent. Different from the initiation latency results, participants took longer to complete their reach movement when they had strong relative to weak temporal expectation about the target onset. Interestingly, these effects interacted such that the delaying effect of response conflict was stronger when combined with strong temporal expectation. This pattern is confirmed by an ANOVA with a significant main effect of congruency, $F(1,17)=15.34, p=.001, \eta_{\mathrm{p}}{ }^{2}=0.47$, a significant main effect of temporal expectation, $F(1,17)=37.44, p$ $<.0001, \eta_{\mathrm{p}}{ }^{2}=0.69$, and a significant interaction effect between them, $F(1,17)=10.07, p=.006, \eta_{\mathrm{p}}{ }^{2}=0.37$. The pairwise comparisons revealed that the congruency effect on movement time was significant for the strong-temporalexpectation trials, $t(17)=4.36, p<.001, d=1.03$, but not for the weak-temporal-expectation trials, $t(17)=1.48, p>.1$ (see Fig. 2b). These results may suggest that temporal expectation prolongs the response conflict resolution process during the reach movement.

Note that longer movement time may reflect a more difficult or effortful process or reduced task focus. The trajectory measures discussed later disambiguate how the distractors influence the reach movement. In particular, we later demonstrate that longer movement times are associated with more curved trajectories toward the incorrect response alternative on incongruent trials.

Lastly, we analyzed total times (see Fig. 2c) measured as the sum of initiation latency and movement time, which can approximate the combination of the response threshold adjustment process and the controlled response selection process, likely reflected by conventional key-press RT. Participants took longer when flankers were incongruent relative to congruent, and when they had weak relative to strong temporal expectation about the target onset, primarily driven by the effects observed on initiation latency. Further, these effects interacted in that the delaying effect of response conflict was stronger when combined with strong temporal expectation, driven by the effects observed on movement time. This pattern is confirmed by an ANOVA with a significant main effect of congruency, $F(1,17)=39.63, p<.0001$, $\eta_{\mathrm{p}}{ }^{2}=0.70$, a significant main effect of temporal expectation, $F(1,17)=24.01, p=.0001, \eta_{\mathrm{p}}{ }^{2}=0.59$, and a significant interaction effect between them, $F(1,17)=7.04, p=.017$, $\eta_{\mathrm{p}}{ }^{2}=.29$. Pairwise comparisons revealed that the congruency effect on total time was present in both the strongtemporal-expectation trials, $t(17)=6.45, p<.0001, d=$ 1.52, and weak-temporal-expectation trials, $t(17)=4.94, p$ $=.0001, d=1.17$ (see Fig. 2c). Nevertheless, the significant interaction effect and the effect size difference indicate that the congruency effect was stronger on the strong-temporalexpectation compared with weak-temporal-expectation trials. Given that total time approximates the key-press RT effects of response conflict, this result may be roughly equivalent to the previous findings showing that the response conflict effect on key-press RT is greater on strong-temporalexpectation relative to weak-temporal-expectation trials.

In summary, temporal expectation speeded the initiation of reach movements regardless of whether there was response conflict or not, suggesting that temporal expectation can adjust the response threshold independently of response conflict. Notably, temporal expectation prolonged the duration of reach movements, especially when there was response conflict. The finding that temporal expectation also prolonged movement times on congruent trials suggests that temporal expectation may lead to more cautious actions followed by fast movement initiation in the absence of a response conflict. The trajectory measures reported in the next section-curvature and distraction attraction score - further examined how temporal expectation modulated the controlled response selection process. 
Evidence from trajectory measures: Curvature and distraction attraction score To investigate how temporal expectation modulated the controlled selection process, we examined the reach trajectories using curvature and distractor attraction score.

Figure 3a shows the group mean trajectories for congruent (solid curve) and incongruent (dashed curve) trials, separately plotted for the correct leftward and rightward responses. Trajectories are further broken down into strong-temporalexpectation (left panel) and weak-temporal-expectation (right panel) trials. As predicted, participants had trajectories that were more curved toward the competing response alternative when flankers were incongruent relative to congruent. This is indicated by the dashed curves (incongruent) being pulled inward compared with the solid curves being relatively straight in Fig. 3a. This finding confirms that competing response alternatives are coactivated during the controlled response selection process when there is response conflict. Notably, the strength of this effect was modulated by temporal expectation such that the trajectories on incongruent trials were more strongly curved toward the incorrect response alternative when participants had strong relative to weak temporal expectation about the target onset. This is indicated by the average negative space between the solid and dashed curves being greater in the left panel compared with the right panel in Fig. 3a.

To quantify our observation and capture the controlled response selection process (Erb \& Marcovitch, 2018; Erb et al., 2016; Erb et al., 2017), we calculated a maximum curvature such that positive values indicated the degree to which the trajectory is curved toward the incorrect response alternative, negative values indicated curvature away from the incorrect response alternative (or toward the correct response alternative), and a value of zero indicated a straight-line trajectory (see Method section). Our observations were confirmed by an ANOVA with a significant main effect of congruency $(M=$ $-0.59, S E=0.29$ for congruent trials vs. $M=4.39, S E=0.86$ for incongruent trials), $F(1,17)=30.98, p<.0001, \eta_{\mathrm{p}}{ }^{2}=.65$, a significant main effect of temporal expectation $(M=2.56, S E$ $=0.63$ for strong-temporal-expectation trials vs. $M=1.24, S E$ $=0.37$ for weak-temporal-expectation trials), $F(1,17)=8.69$, $p=.01, \eta_{\mathrm{p}}{ }^{2}=0.34$, and a significant interaction between them, $F(1,17)=19.26, p<.001, \eta_{\mathrm{p}}{ }^{2}=0.53$. Pairwise comparisons revealed that the congruency effect on maximum curvature was present on both the strong-temporal-expectation trials $(M=5.69, S E=1.10$, for incongruent vs. $M=-0.58, S E=$ 0.39 for congruent, $t(17)=6.31, p<.0001, d=1.49)$ and the weak-temporal-expectation trials $(M=3.08, S E=0.74$ for incongruent vs. $M=-0.60, S E=0.33$ for congruent), $t(17)$ $=4.15, p<.001, d=0.98$. Nevertheless, the significant interaction effect and the effect size difference indicate that the congruency effect was stronger on the strong-temporalexpectation compared with weak-temporal-expectation trials.
These results indicate that temporal expectation modulated the controlled response selection such that it increased the response conflict effect. This is also consistent with the pattern of the movement time results.

We then analyzed the distractor attraction scores (incongruent minus congruent difference trajectories) to examine the continuous effects of temporal expectation on response conflict. Figure $3 \mathrm{~b}$ shows the distractor attraction scores for strong-temporal-expectation (thick curve) and weaktemporal-expectation (thin curve) trials. The effect of temporal expectation on response conflict was evident early on and lasted until over halfway into the reach movement, indicated by the extended difference between thick and thin curves in Fig. 3b. This was confirmed by the point-by-point comparisons of the difference curves. Results revealed that the temporal expectation effect was significant starting at $4 \%$ and lasting until $64 \%$ into the reach movement, corrected using the false discovery rate (FDR) correction according to Benjamini and Hochberg's (1995) method (see asterisks for uncorrected and open circles for corrected significant-difference regions in Fig. $3 b)$. In summary, the trajectory measures provided further evidence suggesting that temporal expectation prolongs the controlled response selection process.

In parallel, we tested whether the reported congruency effect on curvature reflects the degree of activation of the incorrect response as we presumed, or whether it reflects indecision such that participants may start moving toward the center of the display before they reach a decision more so on incongruent trials. To that end, we assessed at what point in the movement the incongruent-trial trajectories diverged from the midline $(x=0)$, and confirmed that both the left-incongruent and right-incongruent trial trajectories statistically diverged from the midline path as early as $1 \%$ into the reach movement, FDR corrected (see dashed lines diverging almost immediately from an imaginary midline and also form each other in Fig. 3a [also Fig. 5a], and the similarity of this pattern between left and right panels). This indicates that the observed congruency effect on curvature more likely reflects the activation of the incorrect response alternative rather than indecision, which would be reflected in incongruent trajectories falling along the midline $(x=0)$ in the beginning of the reach movement (i.e., a period of indecision in the movement).

Note that the abovementioned temporal expectation effects were calculated by comparing the strong-temporalexpectation trials, which are composed of only primedtiming trials, and the weak-temporal-expectation trials, which are composed of both primed-timing and unprimed-timing trials. To better equate the inherent temporal priming effects across the two temporal expectation conditions, we excluded the unprimed-timing trials from analyses and confirmed the same pattern of results. This suggests that the effects of temporal expectation reported above cannot be solely explained by temporal priming differences. 


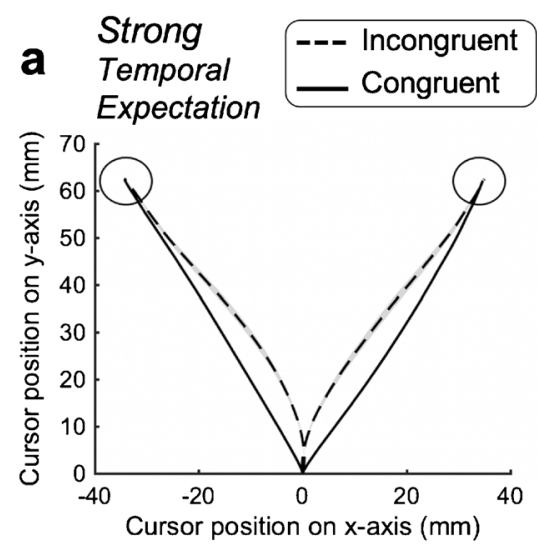

Weak

Temporal

Expectation

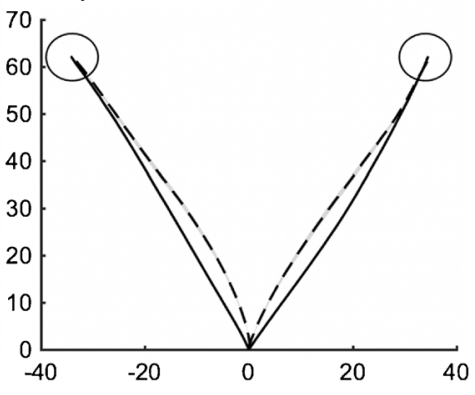

b

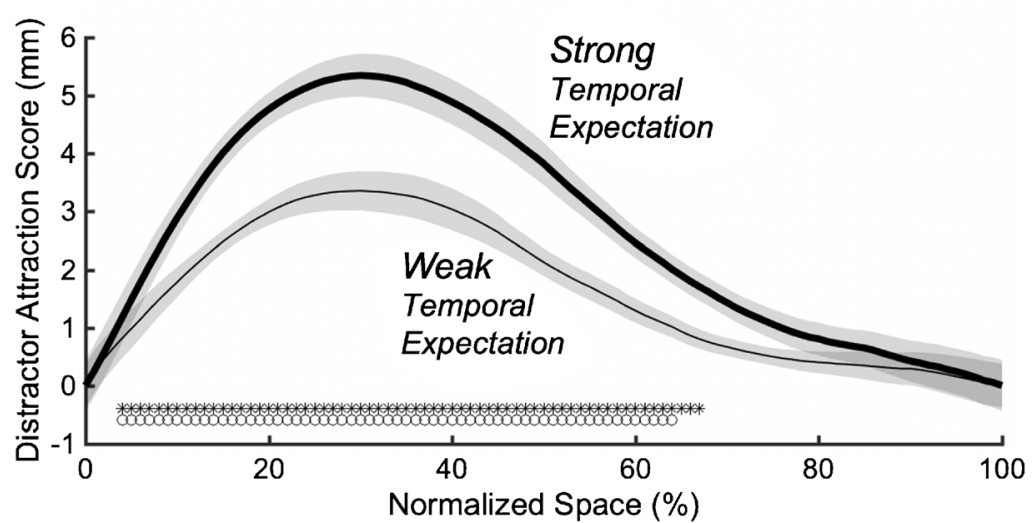

Fig. 3 The effects of temporal expectation and flanker congruency on trajectory curvature and distraction attraction scores are shown. a Group mean cursor trajectories are shown for congruent (solid curve) and incongruent (dashed curve) trials, for correct left and right responses, separately, for strong-temporal-expectation trials on the right and weaktemporal-expectation trials on the left. The point $(0,0)$ indicates the start location and circles indicate the response locations, roughly drawn to scale. Shaded error bands represent \pm 1 regular standard error of the

\section{Temporal priming does not modulate response conflict}

Evidence from chronometric measures: Initiation latency, movement time, and total time On average, task accuracy was relatively high $(M=87.28 \%, S E=2.67 \%)$, and the majority of the errors $(\sim 70 \%)$ were due to missing the time deadline. Participants were more accurate on the primed-timing $(M$ $=89.99 \%, S E=2.37 \%)$ than unprimed-timing trials $(M=$ $85.72 \%, S E=2.96 \%), F(1,17)=8.27, p=.010, \eta_{\mathrm{p}}^{2}=0.33$, as well as on the congruent $(M=90.54 \%, S E=2.22 \%)$ than incongruent trials $(M=85.17 \%, S E=3.17 \%), F(1,17)=8.21$, $p=.011, \eta_{\mathrm{p}}{ }^{2}=0.33$, with no significant interaction effect between temporal priming and congruency on accuracy, $F(1,17)=0.05, p>.8$. This provides no evidence of a speed-accuracy trade-off when the initiation latency effects are considered below. mean - however, they are too small to discern. b Distractor attraction scores (incongruent minus congruent trajectory difference) for strongtemporal-expectation (thick curve) and weak-temporal-expectation (thin curve) trials are shown. Shaded error bands represent \pm 1 standard error of the mean, adjusted for within-participants comparisons (Morey, 2008). Asterisks in the bottom indicate regions of significant differences, uncorrected for multiple comparisons; open circles indicate significant regions that survive the FDR correction 


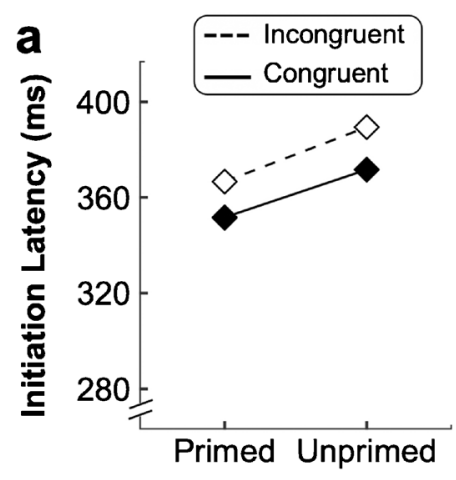

Temporal Priming

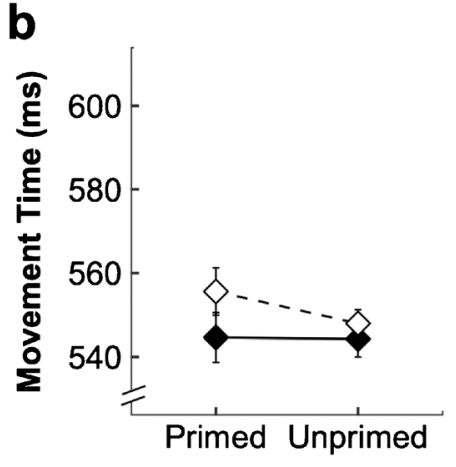

Temporal Priming

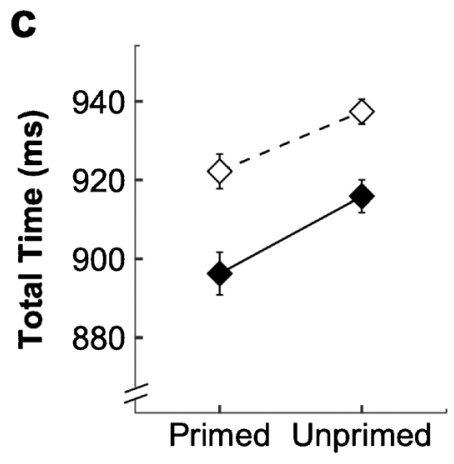

Temporal Priming

Fig. 4 The effects of temporal priming and flanker congruency on (a) initiation latency, (b) movement time, and (c) total time are shown. The error bars represent \pm 1 standard error of the mean, adjusted for within-participants comparisons (Morey, 2008)

priming, similar to temporal expectation, lowers response threshold while response conflict elevates response threshold, independently of one another.

Next, we analyzed the movement time and found that none of the effects were statistically significant, $F(1,17)=2.21, p>$ .10 , for the main effect of congruency, $F(1,17)=0.82, p>.30$, for the main effect of temporal priming, and $F(1,17)=0.47, p>$ .50 , for the interaction effect (see Fig. 4b).

Lastly, we analyzed the total time (sum of initiation latency and movement time), which can capture the combination of the response threshold adjustment and the controlled response selection processes in response conflict resolution. The pattern of results was the same as that for the initiation latency given the null effects on the movement time. Participants took longer when flankers were incongruent relative to congruent, and when the target timing was unprimed relative to primed, in an additive manner (see Fig. 4c). This pattern is confirmed by an ANOVA revealing a significant main effect of congruency, $F(1,17)=21.14, p<.001, \eta_{\mathrm{p}}{ }^{2}=0.55$, a significant main effect of temporal priming, $F(1,17)=26.01, p<.0001, \eta_{\mathrm{p}}{ }^{2}$ $=0.60$, and no interaction effect between them, $F(1,17)=$ $0.26, p>.60$ (see Fig. 4c).

In summary, similar to temporal expectation, temporal priming speeded the initiation of reach movements regardless of whether there was response conflict or not, suggesting that temporal priming can also adjust the response threshold independently of response conflict. Unlike temporal expectation, temporal priming did not affect the duration of reach movements.

\section{Evidence from trajectory measures: Curvature and distractor} attraction score Similar to temporal expectation analyses, we further examined whether and how temporal priming modulated the controlled selection process using the curvature and distractor attraction scores.
Figure 5a shows the group mean trajectories for congruent (solid curve) and incongruent (dashed curve) trials, separately plotted for the correct leftward and rightward responses. The trajectories were further broken down into primed-timing (left panel) and unprimedtiming (right panel) trials. As predicted, participants had trajectories that were more curved toward the competing response alternative when flankers were incongruent relative to congruent, which is indicated by the dashed curves being pulled inward and the solid curves being relatively straight in Fig. 5a. This observation is confirmed by an ANOVA with a main effect of congruency $(M=3.21, S E=0.80$ for incongruent vs. $M=-0.61, S E$ $=0.32$ for congruent trials), $F(1,17)=16.29, p<.001$, $\eta_{\mathrm{p}}^{2}=0.49$. This finding further demonstrates that the competing response alternatives were coactivated during the controlled response selection process when there was response conflict.

However, we observed that the congruency effect did not differ between primed-timing (left panel, congruency effect), $t(17)=3.41, p=.003, d=.80$, and unprimedtiming trials (right panel; congruency effect), $t(17)=$ $4.14, p=.001, d=.97$. This was confirmed by an ANOVA with no main effect of temporal priming, $F(1$, $17)=0.77, p>.30$, or interaction effect, $F(1,17)=1.97$, $p>.10$. These results show that, unlike temporal expectation, temporal priming did not reliably modulate the controlled response selection process beyond a nonsignificant, numeric effect.

We then analyzed the distractor attraction scores (incongruent minus congruent difference trajectories) to examine the continuous effects of temporal priming on response conflict. Figure $5 \mathrm{~b}$ shows the distractor attraction scores for primed-timing (thick curve) and unprimed-timing (thin curve) trials. The point-by-point comparison of the difference curves paralleled the maximum curvature results, 

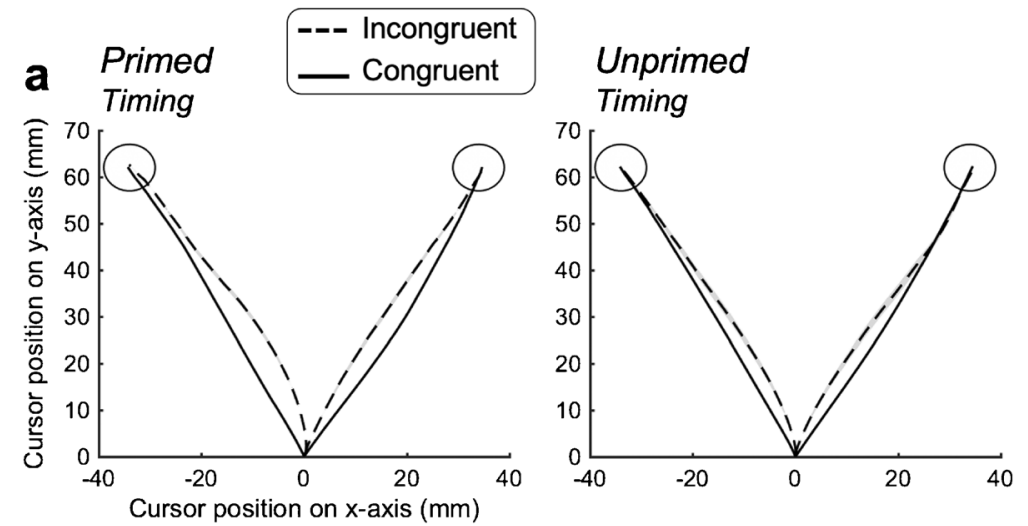

b

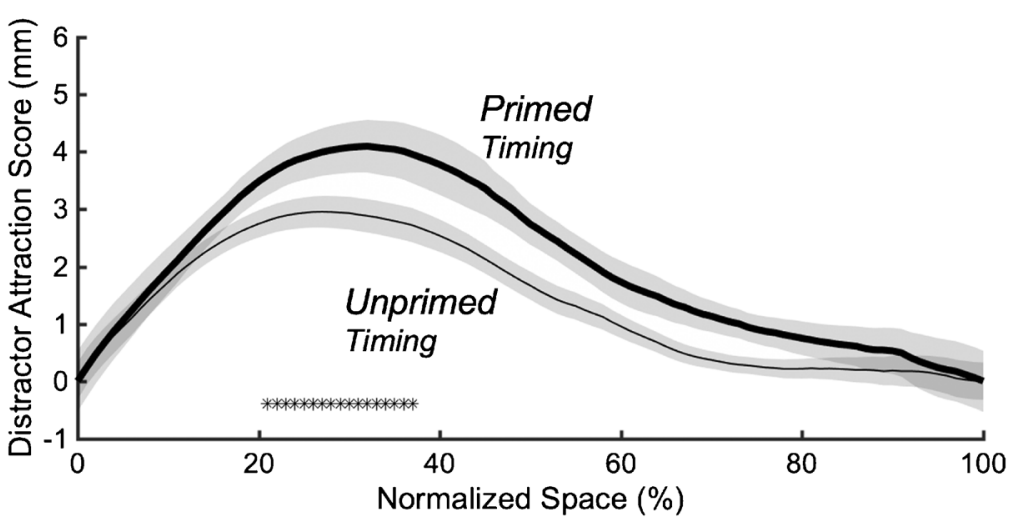

Fig. 5 The effects of temporal priming and flanker congruency on trajectory curvature and distraction attraction scores are shown. a Group mean cursor trajectories are shown for congruent (solid curve) and incongruent (dashed curve) trials, for correct left and right responses, separately for primed-timing trials on the right and unprimed-timing trials on the left. The point $(0,0)$ indicates the start location and circles indicate the response locations, roughly drawn to scale. Shaded error bands represents \pm 1 regular standard error of the mean, however they are too small

revealing no differences that survive the FDR correction (see asterisks for uncorrected significant-difference regions in Fig. 5b).

In summary, the trajectory measures provided little evidence that temporal priming influences the controlled response selection process, unlike temporal expectation. Nevertheless, we observed a small but nonsignificant effect in the same direction as temporal expectation.

Overall, we observed that both temporal expectation and temporal priming lowered the response threshold independently of response conflict that increased the response threshold. However, the effects of temporal expectation and priming on the controlled response selection process diverged. Temporal expectation substantially prolonged the influences of the competing response alternative during the controlled response selection process whereas the effect of temporal priming was unreliable. This may indicate that goal-driven rather than stimulus-driven temporal orienting more reliably prolongs response conflict resolution. to discern. b Distractor attraction scores (incongruent minus congruent trajectory difference) for primed-timing (thick curve) and unprimedtiming (thin curve) trials are shown. Asterisks in the bottom indicate significant regions, uncorrected for multiple comparisons; none of the points survived the FDR correction. Shaded error bands represent \pm 1 standard error of the mean, adjusted for within-participants comparisons (Morey, 2008)

\section{A general trade-off between the response threshold adjustment process and the controlled response selection process during response conflict resolution}

The current framework on response conflict posits that when conflict is detected, the response threshold is adjusted to halt the motor responses so that the controlled response selection process can sufficiently favor the activation of the correct response alternative in a timely manner (Erb \& Marcovitch, 2018; Erb et al., 2020). It follows that if the response threshold is not increased enough (for instance, due to random variability in performance or other factors that can systematically lower response thresholds), the controlled response selection process may be impaired or may need to be prolonged. Consistent with this view, previous studies have reported that shorter movement initiation latencies are coupled with greater curvature reflecting a greater conflict effect using a Simon task (Buetti \& Kerzel, 2008, 2009) as well as in a reaching task with a cluttered visual display (Meegan \& Tipper, 1998). 
Thus, we assessed the potential negative relationship between the response threshold adjustment process and the controlled response selection process.

Using only incongruent trials, we regressed curvature (calculated as maximum curvature in $\mathrm{mm}$ ) on initiation latency $(\mathrm{ms})$ for each participant using all timing trials (including all short, medium, and long interval trials in all blocks) with correct responses. Figure 6a shows a negative correlation between initiation latency and curvature observed in one participant. Figure $6 \mathrm{~b}$ shows the histogram of slopes from all 18 participants. As predicted, slopes were consistently negative across all participants. This was confirmed by a single-sample $t$ test showing that the slopes were significantly different from zero $(M=-0.043$, $S E=0.006), t(17)=6.68, p<.0001$, indicating that on average, 1 -ms decrease in initiation latency predicts $\sim 0.04-\mathrm{mm}$ increase in curvature observed on incongruent trials. In other words, on trials where response threshold was lower (less motor inhibition) at movement initiation, the incorrect response alternative more strongly attracted the cursor trajectory (stronger relative activation of the incongruent response alternative) during movement.

We also confirmed that this relationship was present in a subset of data that did not contribute to the main temporal orienting effects. We repeated the same analysis using only medium-interval and long-interval trials. Similar to the full data set, slopes were consistently negative across participants, $(M=-0.042, S E=0.008), t(17)=4.67, p<.0001$. This further confirms that shorter initiation latencies predict greater curvature on incongruent trials even without the variation in temporal orienting in the data set.

Taken together, these results support the idea that there is a general trade-off between the response threshold adjustment process and the controlled response selection process. Such a compensatory mechanism may help explain the differences between the effects of goal-driven versus stimulus-driven temporal orienting on the curvature. While goal-driven temporal expectation and stimulus-driven temporal priming both lowered response thresholds, temporal expectation had a greater effect ( $73-\mathrm{ms}$ faster on strongtemporal-expectation than weak-temporal-expectation trials) than temporal priming ( $\sim 21-\mathrm{ms}$ faster on primedtiming than unprimed-timing trials) on initiation latencies. Further, temporal expectation significantly increased curvature on incongruent trials $(\sim 2.6 \mathrm{~mm}$ more curvature on strong-temporal-expectation than weak-temporalexpectation trials), whereas temporal priming only had a nonsignificant numeric effect $(\sim 1.2$-mm more curvature on strong-temporal-expectation than weak-temporalexpectation trials). Although purely correlational, one may speculate that goal-driven attentional orienting had a more robust effect on response selection during movement than stimulus-driven orienting potentially because of the different strengths of their influence on response thresholds at movement initiation. However, temporal orienting might instead influence response conflict via its effects on another process (i.e., a third variable).

\section{Discussion}

Temporal orienting enables us to more effectively prepare for an upcoming event, facilitating behavioral responses to that event, indicated by speeded motor responses and/or increased sensitivity (for a review, see Nobre \& van Ede, 2018). While such facilitatory effects are well established for simple tasks involving no conflict, several studies have reported mixed results on the effects of temporal orienting on response conflict using paradigms such as Eriksen's flanker or Simon tasks with key-press RT measures (e.g., Ball et al., 2019; Correa et al., 2010; Korolczuk et al., 2018; Menceloglu et al., 2017). Here, we aimed to understand these heterogeneous findings by potentially revealing the more nuanced effects of both endogenous and exogenous temporal orienting on response conflict using a choice-reaching task. Notably, choice-reaching tasks can reveal the dynamic processes involved in goaldirected action (for a review, see Song \& Nakayama, 2009), such as the response threshold adjustment and controlled response selection during response conflict (Erb \& Marcovitch, 2018; Erb et al., 2016; Erb et al., 2017).

We observed robust flanker effects; participants initiated their reach later, and their reach trajectories were more curved toward the incorrect response alternative on incongruent trials compared with congruent trials. The congruency effect was also reflected in movement times being longer on incongruent trials. These results confirm the effectiveness of our response conflict manipulation. They also replicate the previous findings that the presence of response conflict elevates response thresholds and increases coactivation of the competing response alternatives during the controlled response selection process (e.g., Erb et al., 2016; Erb et al., 2017; Faulkenberry et al., 2016; Scherbaum et al., 2010). We also observed robust temporal orienting effects; participants initiated their reach earlier on trials where they had strong compared with weak temporal expectation about the target onset as well as on trials where target timing was repeated (primed) compared with switched (unprimed). These results confirm the effectiveness of our temporal expectation and temporal priming manipulation, and replicate the previous key-press RT effects of temporal orienting (e.g., Correa et al., 2004; Menceloglu et al., 2019; Mento, 2017).

Further, the effects of flanker congruency and temporal orienting on the initiation latency were additive, suggesting that response conflict and temporal orienting independently contribute to the adjustment of response thresholds. While we observed independent behavioral effects, future research 


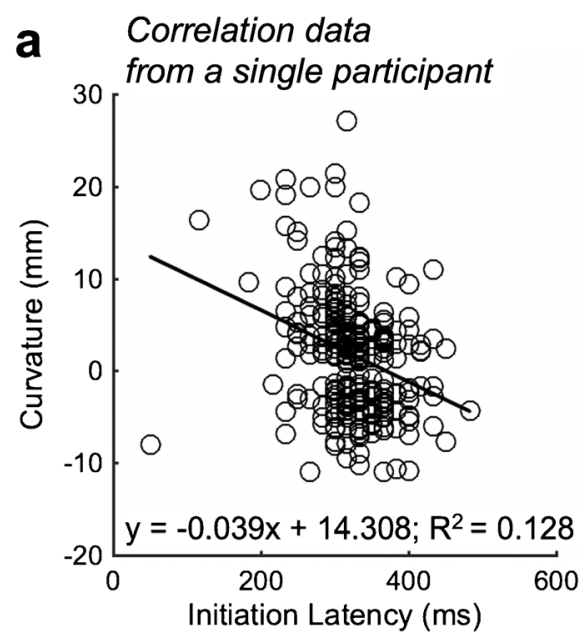

Fig. 6 The negative correlation between initiation latency and curvature on incongruent trials is depicted. a Correlation data from a single participant using all timing trials is plotted, showing that 1-ms decrease in initiation latency predicts a $0.039 \mathrm{~mm}$ increase in curvature on

relying on neural measurements is needed to more conclusively answer whether the adjustments to response threshold via the presence of response conflict (elevating response threshold) and temporal orienting (lowering response threshold) are implemented via distinct or overlapping neural mechanisms.

Notably, temporal expectation modulated the congruency effects on curvature such that the incorrect response alternative attracted the trajectories more strongly when participants had strong temporal expectation about the target onset. In other words, temporal expectation influenced the relative strength of the activation along automatic and the controldemanding pathway during the controlled response selection process, in favor of the automatic pathway. This effect was observable early on and lasted until over halfway into the reach movement, demonstrating that temporal expectation prolongs the controlled response selection process, permitting greater influences of conflicting responses during reaching. Temporal priming, however, did not have any reliable influence on curvature, yet the pattern of results mimicked the temporal expectation effects such that the incorrect response alternative attracted the trajectories more strongly when target timing was primed than unprimed. Taken together, these results suggest that endogenous rather than exogenous temporal orienting can more reliably influence the controlled response selection process, interfering with response conflict resolution during reach movement.

We further demonstrated that the dissociation between temporal expectation and temporal priming was likely due to the different strengths of their effects on the response thresholds. In particular, we found that shorter initiation latencies predicted greater curvature on incongruent trials across all timing trials and independent of the variation in temporal orienting, potentially pointing to a general trade-off between

\section{b Slopes from all participants \\ including all timing trials}

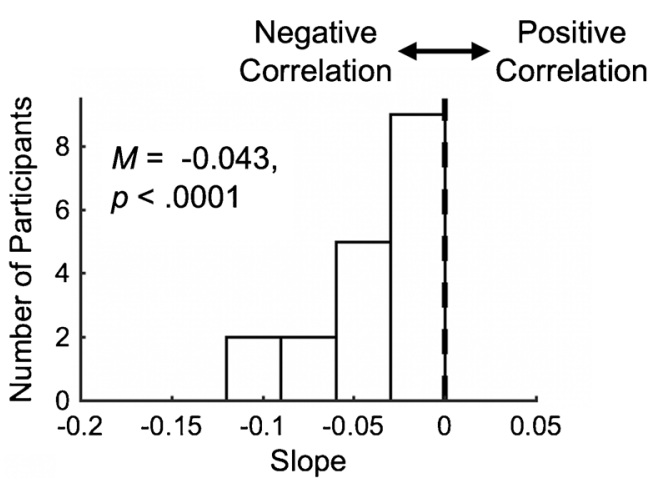

incongruent trials for this participant. b Histogram of slopes from 18 participants using all timing trials is plotted, demonstrating a consistent negative relationship between initiation latency and curvature, across participants

the response threshold adjustment process and the controlled response selection process. This finding, although correlational, suggests that temporal expectation influenced the controlled response selection process indirectly through its effect on response threshold. It also suggests that temporal expectation had a robust effect on response selection during movement, whereas temporal priming did not have a reliable effect, likely because temporal expectation drastically lowered response thresholds while temporal priming had a smaller effect (see Fig. 2a vs. Fig. 4a).

This behavioral dissociation is further supported by neuroimaging studies indicating that cognitive control relies on overlapping neural mechanisms with endogenous rather than exogenous temporal orienting. Indeed, research has consistently shown that temporal expectation relies on prefrontal structures (for a review, see Coull, 2009; Coull, Cheng, \& Meck, 2011) that are also implicated in cognitive control (for a review, see Shenhav et al., 2013), whereas temporal priming does not (e.g., Triviño et al., 2010). In particular, Triviño et al. (2010) measured temporal expectation effects (using block-wise variations in target-timing probability) and temporal priming effects (using immediate target-timing repetition) in prefrontal lesion patients and healthy controls. They reported that patients with prefrontal lesions showed a deficit in temporal expectation effects, but not in temporal priming effects, corroborating the idea that temporal expectation effects involve endogenous, goal-driven orienting of attention, whereas temporal priming effects involve exogenous, stimulus-driven orienting of attention.

Our conclusion that temporal expectation interferes with response conflict potentially through decreasing response inhibition is in line with a recent electromyography (EMG) study by Korolczuk et al. (2020). Korolczuk et al. (2020) 
investigated the effects of temporal expectation on a Simon conflict effect, manipulating temporal expectation using informative cues (100\% valid long or short cue-to-target interval) or neutral cues (equally likely long or short cue-to-target interval) in separate blocks of trials. When only considering average RT, they did not observe temporal cueing effects on the Simon conflict effect. Importantly, however, when considering accuracy as a function of RT, they found that on the incongruent trials, the presence of temporal cues led to fast responses that were more likely to be incorrect or partially incorrect, which was indicated by subthreshold activations (measured by EMG) of the incorrect response hand before a correct key-press response. Further, the latency of the EMGbased partial errors was shorter on trials with temporal cues. Thus, they concluded that temporal orienting could lead to impulsive and premature responses, which can be detrimental when there is response conflict. These findings also indicate that key-press RT alone may not be sensitive enough to reveal the effects of temporal expectation on response conflict, explaining the prior heterogenous key-press RT results.

While reach and key-press tasks are different, as the former allows for online changes to responses while the latter is a ballistic response, one may suspect that key-press RT is the aggregate of the separate measures afforded by reach tracking. Our results may indicate that key-press RT reflects a combination of initiation latency and curvature, which is roughly the total time measure in our experiment. When only considering total time, the effect size of the interaction effect between temporal expectation and congruency is smaller relative to the curvature effect. This may be equivalent to key-press RT studies showing only small interaction effects between temporal expectation and response conflict (e.g., Correa et al., 2010; Korolczuk et al., 2018).

However, key-press RT results may be different depending on the relative modulations of the response threshold adjustment and controlled response selection processes by temporal expectation (see the opposite interaction patterns in Figs. 2a and b). For instance, we found that temporal expectation decreased initiation latency while response conflict increased it. However, a nonsignificant, numeric effect was observed such that response conflict increased initiation latency slightly more on weak-temporal-expectation compared with strongtemporal-expectation trials (see Fig. 2a). In contrast, response conflict increased movement times more on strong-temporalexpectation compared with weak-temporal-expectation trials (see Fig. 2b). Consequently, if comparable, these opposing effects could result in additive temporal expectation and response conflict effects on total time, corresponding to the previously reported key-press RT effects showing no interaction (e.g., Ball et al., 2019; Menceloglu et al., 2017). This approach therefore helps explain the heterogeneous key-press RT findings. Nevertheless, more research is needed to thoroughly characterize the similarities and differences between key- press and reach behavior in terms of response conflict resolution and the more general effects of temporal orienting on motor behavior.

Our conclusion that temporal orienting lowers response threshold in general is supported by neural data demonstrating that temporal orienting modulates the excitability of the motor cortex. For instance, a recent EEG study demonstrated that when the likely key-press response side is associated with a certain cue-to-target interval, the polarity and the amplitude of the lateralized readiness potential (LRP; movement-related, slow-wave ERP) were modulated by the passage of time. This suggests that temporal orienting can modulate the preparation of the effector-specific motor responses (Volberg \& Thomaschke, 2017). Studies have also consistently demonstrated that the amplitude of the contingent negative variation (CNV; a negative-going slow-wave ERP thought to index target anticipation and response preparation) is modulated by temporal expectation, especially in motor tasks (e.g., Correa et al., 2006; Miniussi et al., 1999). In the frequency domain, EEG studies have reported stronger suppression of movement-related beta $(\sim 13-30 \mathrm{~Hz}$ activity observed over frontocentral electrodes) associated with responses to temporally expected or regular targets (e.g., Alegre et al., 2003; Breska \& Deouell, 2016; Praamstra et al., 2006). Further, neuroimaging studies have shown that temporal orienting engages the left intraparietal sulcus (IPS) both for perceptual and motor tasks (e.g., Cotti et al., 2011; Coull \& Nobre, 1998; Davranche et al., 2011), and that the level of left IPS activation correlates with bilateral premotor and motor cortex activation specifically during motor tasks, potentially demonstrating the role of temporal orienting in motor preparation.

Alternatively, the observed effects of temporal orienting on initiation latencies may also reflect adjustments to the evidence accumulation rate. However, a recent study using drift diffusion modeling (Ratcliff \& McKoon, 2008; Ratcliff \& Rouder, 1998) of key-press RT data along with EEG has suggested that temporal expectation speeds decision onset, without affecting evidence quality, in a visual detection task (van den Brink et al., 2020). Nevertheless, future modeling efforts that incorporate the automatic and controlled stimulus processing pathways in conflict tasks (e.g., Diffusion Model for Conflict Tasks [DMC]; Ulrich et al., 2015) as well as neural data that can dissociate decision/response boundaries and evidence accumulation will be needed to understand the mechanisms by which temporal orienting modulates choicereaching behavior under conflict.

Lastly, in the current study, we used $0.6 \mathrm{~s}$ as the duration of the short interval that was either strongly versus weakly expected, or primed versus unprimed. Studies have reported temporal expectation and temporal priming effects comparable with ours using different time intervals, in which the shortest interval could range from a few hundred milliseconds to a few seconds. Nevertheless, further research is needed to 
characterize the potential dependencies of the reported effects on specific time intervals. For instance, temporal orienting effects could diminish if time intervals are made longer thereby making time perception less sensitive (Grondin, 2014), or such effects could be tuned to specific time intervals that are more frequently observed in everyday human action (e.g., $500 \mathrm{~ms}$ as the natural rhythmic tapping interval (Moelants, 2002) or walking (MacDougall \& Moore, 2005).

To conclude, our study shows that temporal orienting leads to increased response readiness. While this increased response readiness is shown to be beneficial in simple tasks with no conflict, it was associated with impaired response conflict resolution in our task. We further show that this effect is driven more reliably by endogenous rather than exogenous temporal orienting mechanisms. Overall, our results highlight the interplay between cognitive control and temporal orienting in goaldirected action, and thus have important implications for design of experimental paradigms and everyday situations where we make timely, and sometimes hasty, decisions among distractions.

Supplementary Information The online version contains supplementary material available at https://doi.org/10.3758/s13414-020-02235-4.

\section{Acknowledgment This study was supported by NSF BCS 1849169 to J.H.S.}

Open practices statement None of the data or materials for the experiment reported here is available online, but the data and materials can be provided upon request. The experiment was not preregistered.

\section{References}

Alegre, M., Gurtubay, I. G., Labarga, A., Iriarte, J., Malanda, A., \& Artieda, J. (2003). Alpha and beta oscillatory changes during stimulus-induced movement paradigms: Effect of stimulus predictability. NeuroReport, 14(3), 381-385. https://doi.org/10.1097/ 00001756-200303030-00017

Ball, F., Groth, R. M., Agostino, C. S., Porcu, E., \& Noesselt, T. (2019). Explicitly versus implicitly driven temporal expectations: No evidence for altered perceptual processing due to top-down modulations. Attention, Perception, \& Psychophysics, 1-15. Advance online publication. https://doi.org/10.3758/s13414-019-01879-1

Benjamini, Y., \& Hochberg, Y. (1995). Controlling the false discovery rate: A practical and powerful approach to multiple testing. Journal of the Royal Statistical Society: Series B (Methodological), 57(1), 289-300.

Brainard, D. H. (1997). The Psychophysics Toolbox. Spatial Vision, 10(4), 433-436. https://doi.org/10.1163/156856897X00357

Breska, A., \& Deouell, L. Y. (2016). When synchronizing to rhythms is not a good thing: Modulations of preparatory and post-target neural activity when shifting attention away from on-beat times of a distracting rhythm. Journal of Neuroscience, 36(27), 7154-7166. https://doi.org/10.1523/JNEUROSCI.4619-15.2016
Buetti, S., \& Kerzel, D. (2008). Time course of the Simon effect in pointing movements for horizontal, vertical, and acoustic stimuli: Evidence for a common mechanism. Acta Psychologica, 129(3), 420-428. https://doi.org/10.1016/j.actpsy.2008.09.007

Buetti, S., \& Kerzel, D. (2009). Conflicts during response selection affect response programming: Reactions toward the source of stimulation. Journal of Experimental Psychology: Human Perception and Performance, 35(3), 816. https://doi.org/10.1037/a0011092

Correa, Á., Lupiáñez, J., Madrid, E., \& Tudela, P. (2006). Temporal attention enhances early visual processing: A review and new evidence from event-related potentials. Brain Research, 1076(1), 116128. https://doi.org/10.1016/j.brainres.2005.11.074

Correa, Á., Lupiáñez, J., Milliken, B., \& Tudela, P. (2004). Endogenous temporal orienting of attention in detection and discrimination tasks. Perception \& Psychophysics, 66(2), 264-278. https://doi.org/10. 3758/BF03194878

Correa, Á., Lupiáñez, J., \& Tudela, P. (2005). Attentional preparation based on temporal expectancy modulates processing at the perceptual level. Psychonomic Bulletin \& Review, 12(2), 328-334. https:// doi.org/10.3758/BF03196380

Correa, Á., Triviño, M., Pérez-Dueñas, C., Acosta, A., \& Lupiáñez, J. (2010). Temporal preparation, response inhibition and impulsivity. Brain and Cognition, 73(3), 222-228. https://doi.org/10.1016/j. bandc.2010.05.006

Cotti, J., Rohenkohl, G., Stokes, M., Nobre, A. C., \& Coull, J. T. (2011). Functionally dissociating temporal and motor components of response preparation in left intraparietal sulcus. NeuroImage, 54(2), 1221-1230. https://doi.org/10.1016/j.neuroimage.2010.09.038

Coull, J. T. (2009). Neural substrates of mounting temporal expectation. PLoS Biology, 7(8), e1000166.

Coull, J. T., \& Nobre, A. C. (1998). Where and when to pay attention: the neural systems for directing attention to spatial locations and to time intervals as revealed by both PET and fMRI. Journal of Neuroscience, 18(18), 7426-7435. https://doi.org/10.1523/ JNEUROSCI.18-18-07426

Coull, J. T., Cheng, R. K., \& Meck, W. H. (2011). Neuroanatomical and neurochemical substrates of timing. Neuropsychopharmacology, 36(1), 3-25.

Davranche, K., Nazarian, B., Vidal, F., \& Coull, J. (2011). Orienting attention in time activates left intraparietal sulcus for both perceptual and motor task goals. Journal of cognitive neuroscience, 23(11), 3318-3330. https://doi.org/10.1162/jocn_a_00030

Desmurget, M., Jordan, M., Prablanc, C., \& Jeannerod, M. (1997). Constrained and unconstrained movements involve different control strategies. Journal of Neurophysiology, 77(3), 1644-1650. https:// doi.org/10.1152/jn.1997.77.3.1644

Dotan, D., Pinheiro-Chagas, P., Al Roumi, F., \& Dehaene, S. (2019). Track it to crack it: Dissecting processing stages with finger tracking. Trends in Cognitive Sciences, 23(12), 1058-1070. https://doi. org/10.1016/j.tics.2019.10.002

Erb, C. D., \& Marcovitch, S. (2018). Deconstructing the Gratton effect: Targeting dissociable trial sequence effects in children, pre-adolescents, and adults. Cognition, 179, 150-162.

Erb, C. D., Moher, J., Sobel, D. M., \& Song, J. H. (2016). Reach tracking reveals dissociable processes underlying cognitive control. Cognition, 152, 114-126. https://doi.org/10.1016/j.cognition.2016. 03.015

Erb, C. D., Moher, J., Song, J. H., \& Sobel, D. M. (2017). Cognitive control in action: Tracking the dynamics of rule switching in 5-to 8year-olds and adults. Cognition, 164, 163-173. https://doi.org/10. 1016/j.cognition.2017.03.023

Erb, C. D., Smith, K. A., \& Moher, J. (2020). Tracking continuities in the flanker task: From continuous flow to movement trajectories. Attention, Perception, \& Psychophysics, 1-17. Advance online publication. https://doi.org/10.3758/s13414-020-02154-4 
Eriksen, B. A., \& Eriksen, C. W. (1974). Effects of noise letters upon the identification of a target letter in a nonsearch task. Perception \& Psychophysics, 16(1), 143-149. https://doi.org/10.3758/ BF03203267

Faulkenberry, T. J., Cruise, A., Lavro, D., \& Shaki, S. (2016). Response trajectories capture the continuous dynamics of the size congruity effect. Acta Psychologica, 163, 114-123.

Gallivan, J. P., \& Chapman, C. S. (2014). Three-dimensional reach trajectories as a probe of real-time decision-making between multiple competing targets. Frontiers in Neuroscience, 8, 215. https://doi. org/10.3389/fnins.2014.00215

Grondin, S. (2014). About the (non)scalar property for time perception. In H. Merchant \& V. de Lafuente (Eds.), Neurobiology of interval timing (Vol. 829, pp. 17-32). Springer. New York. https://doi.org/ $10.1007 / 978-1-4939-1782-22$

Incera, S., \& McLennan, C. T. (2018). The time course of within and between-language interference in bilinguals. International Journal of Bilingualism, 22(1), 88-99. https://doi.org/10.1177/ 1367006916644688

Kleiner, M., Brainard, D., \& Pelli, D. (2007). What's new in Psychtoolbox-3?. Perception, 36(14), 1-16. https://doi.org/10. 1068/v070821

Korolczuk, I., Burle, B., \& Coull, J. T. (2018). The costs and benefits of temporal predictability: impaired inhibition of prepotent responses accompanies increased activation of task-relevant responses. Cognition, 179, 102-110. https://doi.org/10.1016/j.cognition.2018. 06.006

Korolczuk, I., Burle, B., Coull, J. T., \& Smigasiewicz, K. (2020). Mechanisms of impulsive responding to temporally predictable events as revealed by electromyography. Neuroscience, 428, 13 22. https://doi.org/10.1016/j.neuroscience.2019.12.022

Los, S. A. (2010). Foreperiod and sequential effects: Theory and data. Attention and Time, 289(302), 24. https://oi.org/10.1093/acprof: oso/9780199563456.003.0021

Los, S. A., \& Van den Heuvel, C. E. (2001). Intentional and unintentional contributions to nonspecific preparation during reaction time foreperiods. Journal of Experimental Psychology: Human Perception and Performance, 27(2), 370. https://doi.org/10.1037// 0096-1523.27.2.370

MacDougall, H. G., \& Moore, S. T. (2005). Marching to the beat of the same drummer: the spontaneous tempo of human locomotion. Journal of applied physiology, 99(3), 1164-1173. https://doi.org/ 10.1152/japplphysiol.00138.2005

Meegan, D. V., \& Tipper, S. P. (1998). Reaching into cluttered visual environments: Spatial and temporal influences of distracting objects. Quarterly Journal of Experimental Psychology: Section A, Human Experimental Psychology, 51(2), 225-250. https://doi.org/10.1080/ 02724989843000004

Menceloglu, M., Grabowecky, M., \& Suzuki, S. (2017). Comparing the effects of implicit and explicit temporal expectation on choice response time and response conflict. Attention, Perception, \& Psychophysics, 79(1), 169-179. https://doi.org/10.3758/s13414016-1230-4

Menceloglu, M., Grabowecky, M., \& Suzuki, S. (2019). Probabilitydriven and stimulus-driven orienting of attention to time and sensory modality. Attention, Perception, \& Psychophysics, 81(8), 27322744. https://doi.org/10.3758/s13414-019-01798-1

Mento, G. (2017). The role of the P3 and CNV components in voluntary and automatic temporal orienting: A high spatial-resolution ERP study. Neuropsychologia, 107, 31-40. https://doi.org/10.1016/j. neuropsychologia.2017.10.037

Miniussi, C., Wilding, E. L., Coull, J. T., \& Nobre, A. C. (1999). Orienting attention in time: Modulation of brain potentials. Brain, 122(8), 1507-1518. https://doi.org/10.1093/brain/122.8.1507

Moelants, D. (2002, July). Preferred tempo reconsidered. In C. Stevens, D. Burnham, G. McPherson, E. Schubert, \& J. Renwick (Eds.).
Proceedings of the 7th international conference on music perception and cognition (Vol. 2002, pp. 1-4). Adelaide: Causal Productions.

Moher, J., Anderson, B. A., \& Song, J. H. (2015). Dissociable effects of salience on attention and goal-directed action. Current Biology, 25(15), 2040-2046.

Moher, J., Sit, J., \& Song, J. H. (2015). Goal-directed action is automatically biased towards looming motion. Vision Research, 113, 188197. https://doi.org/10.1016/j.visres.2014.08.005

Moher, J., \& Song, J. H. (2013). Context-dependent sequential effects of target selection for action. Journal of Vision, 13(8), 10-10. https:// doi.org/10.1167/13.8.10

Moher, J., \& Song, J. H. (2019). A comparison of simple movement behaviors across three different devices. Attention, Perception, \& Psychophysics, 81(7), 2558-2569. https://doi.org/10.3758/s13414019-01856-8

Morey, R. D. (2008). Confidence intervals from normalized data: A correction to Cousineau (2005). Reason, 4(2), 61-64. https://doi.org/ 10.20982/tqmp.04.2.p061

Nobre, A. C., Correa, A., \& Coull, J. T. (2007). The hazards of time. Current Opinion in Neurobiology, 17(4), 465-470.

Nobre, A. C., \& van Ede, F. (2018). Anticipated moments: temporal structure in attention. Nature Reviews Neuroscience, 19(1), 34. https://doi.org/10.1038/nrn.2017.141

Pelli, D. G. (1997). The VideoToolbox software for visual psychophysics: Transforming numbers into movies. Spatial Vision, 10(4), 437442. https://doi.org/10.1163/156856897X00366

Praamstra, P., Kourtis, D., Kwok, H. F., \& Oostenveld, R. (2006). Neurophysiology of implicit timing in serial choice reaction-time performance. Journal of Neuroscience, 26(20), 5448-5455. https:// doi.org/10.1523/JNEUROSCI.0440-06.2006

Ratcliff, R., \& McKoon, G. (2008). The diffusion decision model: theory and data for two-choice decision tasks. Neural Computation, 20(4), 873-922. https://doi.org/10.1162/neco.2008.12-06-420

Ratcliff, R., \& Rouder, J. N. (1998). Modeling response times for twochoice decisions. Psychological Science, 9(5), 347-356. https://doi. org/10.1111/1467-9280.00067

Ridderinkhof, K. R., van der Molen, M. W., \& Bashore, T. R. (1995). Limits on the application of additive factors logic: Violations of stage robustness suggest a dual-process architecture to explain flanker effects on target processing. Acta Psychologica, 90(1/3), 29-48. https://doi.org/10.1016/0001-6918(95)00031-O

Ridderinkhof, K. R., Wylie, S. A., van den Wildenberg, W. P., Bashore, T. R., \& van der Molen, M. W. (2020). The arrow of time: Advancing insights into action control from the arrow version of the Eriksen flanker task. Attention, Perception, \& Psychophysics, 1-22. Advance online publication. https://doi.org/10.3758/s13414 $020-02167-z$

Rohenkohl, G., Cravo, A. M., Wyart, V., \& Nobre, A. C. (2012). Temporal expectation improves the quality of sensory information. Journal of Neuroscience, 32(24), 8424-8428. https://doi.org/10. 1523/JNEUROSCI.0804-12.2012

Scherbaum, S., Dshemuchadse, M., Fischer, R., \& Goschke, T. (2010). How decisions evolve: The temporal dynamics of action selection. Cognition, 115(3), 407-416. https://doi.org/10.1016/j.cognition. 2010.02.004

Shenhav, A., Botvinick, M. M., \& Cohen, J. D. (2013). The expected value of control: An integrative theory of anterior cingulate cortex function. Neuron, 79(2), 217-240. https://doi.org/10.1016/j.neuron. 2013.07.007

Simon, J. R., \& Wolf, J. D. (1963). Choice reaction time as a function of angular stimulus-response correspondence and age. Ergonomics, 6(1), 99-105. https://doi.org/10.1080/00140136308930679

Song, J. H. (2017). Abandoning and modifying one action plan for alternatives. Philosophical Transactions of the Royal Society B: Biological Sciences, 372(1718), Article 20160195. https://doi.org/ 10.1098/rstb.2016.0195 
Song, J. H., \& Nakayama, K. (2006). Role of focal attention on latencies and trajectories of visually guided manual pointing. Journal of Vision, 6(9), 11-11. https://doi.org/10.1167/6.9.11

Song, J. H., \& Nakayama, K. (2009). Hidden cognitive states revealed in choice reaching tasks. Trends in Cognitive Sciences, 13(8), 360 366. https://doi.org/10.1016/j.tics.2009.04.009

Spivey, M. J., Grosjean, M., \& Knoblich, G. (2005). Continuous attraction toward phonological competitors. Proceedings of the National Academy of Sciences of the United States of America, 102(29), 10393-10398. https://doi.org/10.1073/pnas.0503903102

Steinborn, M. B., Rolke, B., Bratzke, D., \& Ulrich, R. (2008). Sequential effects within a short foreperiod context: Evidence for the conditioning account of temporal preparation. Acta Psychologica, 129(2), 297-307. https://doi.org/10.1016/j.actpsy.2008.08.005

Triviño, M., Correa, Á., Arnedo, M., \& Lupiáñez, J. (2010). Temporal orienting deficit after prefrontal damage. Brain, 133(4), 1173-1185. https://doi.org/10.1093/brain/awp346

Ulrich, R., Schröter, H., Leuthold, H., \& Birngruber, T. (2015). Automatic and controlled stimulus processing in conflict tasks: Superimposed diffusion processes and delta functions. Cognitive Psychology, 78, 148-174. https://doi.org/10.1016/j.cogpsych.2015. 02.005
Vallesi, A., \& Shallice, T. (2007). Developmental dissociations of preparation over time: Deconstructing the variable foreperiod phenomena. Journal of Experimental Psychology: Human Perception and Performance, 33(6), 1377. https://doi.org/10.1037/0096-1523.33. 6.1377

van den Brink, R. L., Murphy, P. R., Desender, K., de Ru, N., \& Nieuwenhuis, S. (2020). Temporal expectation hastens decision onset but does not affect evidence quality. Journal of Neuroscience. Advance online publication. https://doi.org/10.1523/JNEUROSCI. 1103-20.2020

Vangkilde, S., Coull, J. T., \& Bundesen, C. (2012). Great expectations: temporal expectation modulates perceptual processing speed. Journal of Experimental Psychology: Human Perception and Performance, 38(5), 1183. https://doi.org/10.1037/a0026343

Volberg, G., \& Thomaschke, R. (2017). Time-based expectations entail preparatory motor activity. Cortex, 92, 261-270. https://doi.org/10. 1016/j.cortex.2017.04.019

Publisher's note Springer Nature remains neutral with regard to jurisdictional claims in published maps and institutional affiliations. 